\title{
Compact global attractors of discrete inclusions
}

\author{
David Cheban $^{\mathrm{a}, *}$, Cristiana Mammana ${ }^{\mathrm{b}}$ \\ ${ }^{a}$ State University of Moldova, Department of Mathematics and Informatics, A. Mateevich Street 60, \\ MD-2009 Chişinău, Republic of Moldova \\ ${ }^{\mathrm{b}}$ Institute of Economics and Finance, University of Macerata, Street Crescimbeni 14, I-62100 Macerata, Italy
}

Received 18 August 2005; accepted 26 October 2005

\begin{abstract}
The paper is dedicated to the study of the problem of the existence of compact global attractors of discrete inclusions and to the description of its structure. We consider a family of continuous mappings of a metric space $W$ into itself, and $\left(W, f_{i}\right)_{i \in I}$ is the family of discrete dynamical systems. On the metric space $W$ we consider a discrete inclusion

$$
u_{t+1} \in F\left(u_{t}\right)
$$

associated with $\mathcal{M}:=\left\{f_{i}: i \in I\right\}$, where $F(u)=\{f(u): f \in \mathcal{M}\}$ for all $u \in W$. We give sufficient conditions (the family of maps $\mathcal{M}$ is contracting in the extended sense) for the existence of a compact global attractor of (1). If the family $\mathcal{M}$ consists of a finite number of maps, then the corresponding compact global attractor is chaotic. We study this problem in the framework of non-autonomous dynamical systems (cocyles).

(c) 2005 Elsevier Ltd. All rights reserved.
\end{abstract}

MSC: 37B25; 37B55; 39A11; 39C10; 39C55

Keywords: Global attractor; Set-valued dynamical system; Control system; Chaotic attractor; Collage; Cocycle

\section{Introduction}

The aim of this paper is the study of the problem of the existence of compact global attractors of discrete inclusions and control systems (see, for example, Bobylev et al. [6], Bobylev et al. [7], Emel'yanov et al. [18] and the references therein). Let $W$ be a metric space, $\mathcal{M}:=\left\{f_{i}: i \in I\right\}$

\footnotetext{
* Corresponding author.

E-mail addresses: cheban@usm.md (D. Cheban), cmamman@tin.it (C. Mammana).
} 
be a family of continuous mappings of $W$ into itself and $\left(W, f_{i}\right)_{i \in I}$ be the family of discrete dynamical systems, where $(W, f)$ is a discrete dynamical system generated by positive powers of a continuous map $f: W \rightarrow W$. On the space $W$ we consider a discrete inclusion

$$
u_{t+1} \in F\left(u_{t}\right)
$$

associated with $\mathcal{M}:=\left\{f_{i}: i \in I\right\}(D I(\mathcal{M}))$, where $F(u)=\{f(u): f \in \mathcal{M}\}$ for all $u \in W$.

A solution of the discrete inclusion $D I(\mathcal{M})$ is (see, for example, [6,18,22]) a sequence $\left\{\left\{x_{j}\right\} \mid j \geq 0\right\} \subset W$ such that

$$
x_{j}=f_{i_{j}} x_{j-1}
$$

for some $f_{i_{j}} \in \mathcal{M}$ (trajectory of $D I(\mathcal{M})$ ), i.e.

$$
x_{j}=f_{i_{j}} f_{i_{j-1}} \ldots f_{i_{1}} x_{0} \quad \text { all } f_{i_{k}} \in \mathcal{M} .
$$

We can consider it to be a discrete control problem, where at each moment of time $j$ we can apply a control from the set $\mathcal{M}$, and $D I(\mathcal{M})$ is the set of possible trajectories of the system.

The problem of the existence of compact global attractors for a discrete inclusion arises in a number of different areas of mathematics: control theory - Bobylev et al. [6], Bobylev et al. [7], Emel'yanov et al. [18], Molchanov [26]; linear algebra - Artzrouni [2], Beyn and Elsner [5], Bru et al. [10], Cheban and Mammana [13,14], Daubechies and Lagarias [15], Elsner and Friedland [16], Elsner et al. [17], Gurvits [22], Kozyakin [25], Vladimirov et al. [34], Wirth [36, 37]; Markov chains - Gurvits [19], Gurvits and Zaharin [20,21]; iteration processes — Bru et al. [10], Opoitsev [27]; the Barnsley-Sloan method of fractal image compression — Barnsley and Sloan [4], Bondarenko and Dolnikov [8] and see also the bibliography therein.

In 1988 Barnsley and Sloan [4] put forward some ideas, based on concepts of the theory of dynamical systems, for the compression and storage of graphical information. Their method was called the method of fractal compression of information. They consider a finite set of affine transformations

$$
\mathcal{M}=\left\{f_{i}: i=1,2, \ldots, m\right\}
$$

$\left(f_{i}: \mathbb{R}^{d} \rightarrow \mathbb{R}^{d}\right)$, i.e. transformations $f_{i}$ of the form $f_{i}(u):=A_{i} u+b_{i}$, where $A_{i}$ are square matrices of order $d$, and $u, b_{i} \in \mathbb{R}^{d}$. These transformations possesses the following properties:

(i) there exists a compact $M_{0} \subseteq \mathbb{R}^{d}$ such that $f_{i}(M) \subseteq M_{0}$ for all $M \in K\left(M_{0}\right)$, where $K\left(M_{0}\right)$ is the set of all compact subsets of $M_{0}$;

(ii) $\left|f_{i}\left(u_{1}\right)-f_{i}\left(u_{2}\right)\right| \leq k_{i}\left|u_{1}-u_{2}\right|$, where $k_{i} \in[0,1)$ and $|\cdot|$ is the norm on $\mathbb{R}^{d}$.

This set $\mathcal{M}$ is called an affine collage.

The mapping $F: K\left(M_{0}\right) \rightarrow K\left(M_{0}\right)$ defined by the equality

$$
F(M):=\bigcup_{i=1}^{m} f_{i}(M)
$$

is called a collage mapping.

We consider an arbitrary collage $\mathcal{M}(\mathcal{M}$ contains, generally speaking, an infinite number of mappings $f$ ) on the complete metric space $W$ ( $W$ is not obligatory compact) and we give conditions which guarantee the existence of a compact global attractor for $\mathcal{M}$. If $\mathcal{M}$ consists of a finite number of maps, then we prove that $\mathcal{M}$ admits a compact global chaotic attractor. We study this problem in the framework of non-autonomous dynamical systems (cocyles). 
This paper is organized as follows.

In Section 2 we give some notions and facts from the theory of set-valued dynamical systems which we use in our paper.

Section 3 is dedicated to the study of upper semi-continuous (generally speaking set-valued) invariant sections of non-autonomous dynamical systems. They play a very important role in the study of non-autonomous dynamical systems. We give sufficient conditions guaranteeing the existence of a unique globally exponentially stable invariant section (Theorem 3.2 - the main result of the paper). Analogous statements for non-autonomous dynamical systems, when the base dynamical system $\left(Y, \mathbb{T}_{2}, \sigma\right)$ is invertible, are known (see, for example, [12, Ch.2] and [33]). For the case of the semi-group dynamical system $\left(Y, \mathbb{T}_{2}, \sigma\right)$ (i.e. where the mapping $\sigma(t, \cdot): Y \mapsto Y$ is not invertible) Theorem 3.2 is formulated and proved for the first time in this paper.

In Section 4 we give a new approach to the study of discrete inclusions $(D I)$ which is based on non-autonomous dynamical systems (cocycles). We show that every DI generates in a natural way some non-autonomous dynamical system (cocycle), which plays an important role in its study (see Sections 5 and 6).

Section 5 is dedicated to the study of the relation between the compact global attractor of the cocycle and the skew-product dynamical system (or set-valued dynamical system) associated with the given cocycle (see Theorem 6.2).

In Section 6 we prove that $D I$ generated by a finite number of continuous mappings $f_{1}, f_{2}, \ldots, f_{m}(m \geq 2)$ admits a compact global chaotic attractor.

\section{Set-valued dynamical systems and their compact global attractors}

Let $(X, \rho)$ be a complete metric space, $\mathbb{S}$ be a group of real $(\mathbb{R})$ or integer $(\mathbb{Z})$ numbers, $\mathbb{T}$ $\left(\mathbb{S}_{+} \subseteq \mathbb{T}\right)$ be a semi-group of additive group $\mathbb{S}$. If $A \subseteq X$ and $x \in X$, then we denote by $\rho(x, A)$ the distance from the point $x$ to the set $A$, i.e. $\rho(x, A)=\inf \{\rho(x, a): a \in A\}$. We denote by $B(A, \varepsilon)$ an $\varepsilon$-neighborhood of the set $A$, i.e. $B(A, \varepsilon)=\{x \in X: \rho(x, A)<\varepsilon\}$, and we denote by $K(X)$ the family of all non-empty compact subsets of $X$. With every point $x \in X$ and number $t \in \mathbb{T}$ we associate a closed compact subset $\pi(t, x) \in K(X)$. So, if $\pi(P, A)=\bigcup\{\pi(t, x): t \in P, x \in A\}(P \subseteq \mathbb{T})$, then

(i) $\pi(0, x)=x$ for all $x \in X$;

(ii) $\pi\left(t_{2}, \pi\left(t_{1}, x\right)\right)=\pi\left(t_{1}+t_{2}, x\right)$ for all $x \in X$;

(iii) $\lim _{x \rightarrow x_{0}, t \rightarrow t_{0}} \beta\left(\pi(t, x), \pi\left(t_{0}, x_{0}\right)\right)=0$ for all $x_{0} \in X$ and $t_{0} \in \mathbb{T}$, where $\beta(A, B)=$ $\sup \{\rho(a, B): a \in A\}$ is a semi-deviation of the set $A \subseteq X$ from the set $B \subseteq X$.

In this case it is said [31] that a set-valued semi-group dynamical system is defined.

Let $\mathbb{T}=\mathbb{S}$ and let the following condition be fulfilled:

(i) if $p \in \pi(t, x)$, then $x \in \pi(-t, p)$ for all $x, p \in X$ and $t \in \mathbb{T}$.

Then it is said that a set-valued group dynamical system $(X, \mathbb{T}, \pi)$ or a bilateral (two-sided) dynamical system is defined.

Let $\mathbb{T}^{\prime} \subset \mathbb{S}\left(\mathbb{T} \subset \mathbb{T}^{\prime}\right)$. A continuous mapping $\gamma_{x}: \mathbb{T} \rightarrow X$ is called a motion of the set-valued dynamical system $(X, \mathbb{T}, \pi)$ issuing from the point $x \in X$ at the initial moment $t=0$ and defined on $\mathbb{T}^{\prime}$, if

a. $\gamma_{x}(0)=x$

b. $\gamma_{x}\left(t_{2}\right) \in \pi\left(t_{2}-t_{1}, \gamma_{x}\left(t_{1}\right)\right)$ for all $t_{1}, t_{2} \in \mathbb{T}^{\prime}\left(t_{2}>t_{1}\right)$. 
The set of all motions of $(X, \mathbb{T}, \pi)$, passing through the point $x$ at the initial moment $t=0$ is denoted by $\mathcal{F}_{x}(\pi)$ and $\mathcal{F}(\pi):=\bigcup\left\{\mathcal{F}_{x}(\pi) \mid x \in X\right\}$ (or simply $\mathcal{F}$ ).

The trajectory $\gamma \in \mathcal{F}(\pi)$ defined on $\mathbb{S}$ is called a full (entire) trajectory of the dynamical $\operatorname{system}(X, \mathbb{T}, \pi)$.

Denote by $\Phi(\pi)$ the set of all full trajectories of the dynamical system $(X, \mathbb{T}, \pi)$ and $\Phi_{x}(\pi):=\mathcal{F}_{x}(\pi) \bigcap \Phi(\pi)$.

Theorem 2.1 ([31]). Let $(X, \mathbb{T}, \pi)$ be a semi-group dynamical system and $X$ be a compact and invariant set (i.e. $\pi^{t} X=X$ for all $t \in \mathbb{T}$, where $\left.\pi^{t}:=\pi(t, \cdot)\right)$. Then:

(i) $\mathcal{F}(\pi)=\Phi(\pi)$, i.e. every motion $\gamma \in \mathcal{F}_{x}(\pi)$ can be extended on $\mathbb{S}$ (this means that there exists $\tilde{\gamma} \in \Phi_{x}(\pi)$ such that $\tilde{\gamma}(t)=\gamma(t)$ for all $\left.t \in \mathbb{T}\right)$;

(ii) there exists a group (generally speaking set-valued) dynamical system $(X, \mathbb{S}, \tilde{\pi})$ such that $\left.\tilde{\pi}\right|_{\mathbb{T} \times X}=\pi$.

A system $(X, \mathbb{T}, \pi)$ is called $[11,12]$ compactly dissipative if there exists a non-empty compact $K \subseteq X$ such that

$$
\lim _{t \rightarrow+\infty} \beta\left(\pi^{t} M, K\right)=0 ;
$$

for all $M \in K(X)$.

Let $(X, \mathbb{T}, \pi)$ be compactly dissipative and let $K$ be a compact set attracting every compact subset of $X$. Let us set

$$
J:=\omega(K):=\bigcap_{t \geq 0} \overline{\bigcup_{\tau \geq t} \pi^{\tau} K} .
$$

It can be shown $[11,12]$ that the set $J$ defined by equality (2) does not depend on the choice of the attractor $K$, but is characterized only by the properties of the dynamical system $(X, \mathbb{T}, \pi)$ itself. The set $J$ is called a center of Levinson of the compact dissipative system $(X, \mathbb{T}, \pi)$.

Theorem $2.2([11,12])$. If $(X, \mathbb{T}, \pi)$ is a compactly dissipative dynamical system and $J$ is its center of Levinson, then:

(i) $J$ is invariant, i.e. $\pi^{t} J=J$ for all $t \in \mathbb{T}$;

(ii) $J$ is orbitally stable, i.e. for any $\varepsilon>0$ there exists $\delta(\varepsilon)>0$ such that $\rho(x, J)<\delta$ implies $\beta(x t, J)<\varepsilon$ for all $t \geq 0$;

(iii) $J$ is an attractor of the family of all compact subsets of $X$;

(iv) $J$ is the maximal compact invariant set of $(X, \mathbb{T}, \pi)$.

\section{Continuous invariant sections of non-autonomous dynamical systems}

Let $X$ be a metric space and $Y$ be a topological space. The set-valued mapping $\gamma: Y \rightarrow K(X)$ is said to be upper semi-continuous (or $\beta$-continuous) if $\lim _{y \rightarrow y_{0}} \beta\left(\gamma(y), \gamma\left(y_{0}\right)\right)=0$ for all $y_{0} \in Y$.

Let $(X, h, Y)$ be a fibre bundle [9,23]. The mapping $\gamma: Y \rightarrow K(X)$ is called a section (selector) of the fibre bundle $(X, h, Y)$, if $h(\gamma(y))=y$ for all $y \in Y$.

Remark 3.1. Let $X:=W \times Y$. Then $\gamma: Y \rightarrow X$ is a section of the fibre bundle $(X, h, Y)$ $\left(h:=p r_{2}: X \rightarrow Y\right)$ if and only if $\gamma=\left(\psi, I d_{Y}\right)$ where $\psi: W \rightarrow K(W)$. 
Let $\left(X, \mathbb{T}_{1}, \pi\right)$ and $\left(Y, \mathbb{T}_{2}, \sigma\right)\left(\mathbb{S}_{+} \subseteq \mathbb{T}_{1} \subseteq \mathbb{T}_{2} \subseteq \mathbb{S}\right)$ be two dynamical systems. The mapping $h: X \rightarrow Y$ is called a homomorphism (respectively isomorphism) of the dynamical system $\left(X, \mathbb{T}_{1}, \pi\right)$ on $\left(Y, \mathbb{T}_{2}, \sigma\right)$, if the mapping $h$ is continuous (respectively homeomorphic) and $h(\pi(x, t))=\sigma(h(x), t)\left(t \in \mathbb{T}_{1}, x \in X\right)$.

A triplet $\left\langle\left(X, \mathbb{T}_{1}, \pi\right),\left(Y, \mathbb{T}_{2}, \sigma\right), h\right\rangle$, where $h$ is a homomorphism of $\left(X, \mathbb{T}_{1}, \pi\right)$ on $\left(Y, \mathbb{T}_{2}, \sigma\right)$ and $(X, h, Y)$ is a locally trivial fibre bundle [9,23], is called a non-autonomous dynamical system.

A mapping $\gamma: Y \rightarrow X$ is called an invariant section of the non-autonomous dynamical system $\left\langle\left(X, \mathbb{T}_{1}, \pi\right),\left(Y, \mathbb{T}_{2}, \sigma\right), h\right\rangle$ if it is a section of the fibre bundle $(X, h, Y)$ and $\gamma(Y)$ is an invariant subset of the dynamical system $(X, \mathbb{T}, \pi)$ (or, equivalently,

$$
\bigcup\left\{\pi^{t} \gamma(q): q \in\left(\sigma^{t}\right)^{-1}\left(\sigma^{t} y\right)\right\}=\gamma\left(\sigma^{t} y\right)
$$

for all $t \in \mathbb{T}$ nd $y \in Y$ ).

Theorem 3.2. Let $\left\langle\left(X, \mathbb{T}_{1}, \pi\right),\left(Y, \mathbb{T}_{2}, \sigma\right), h\right\rangle$ be a non-autonomous dynamical system and the following conditions be fulfilled:

(i) the space $Y$ is compact;

(ii) $Y$ is invariant, i.e. $\sigma^{t} Y=Y$ for all $t \in \mathbb{T}_{2}$;

(iii) the non-autonomous dynamical system $\left\langle\left(X, \mathbb{T}_{1}, \pi\right),\left(Y, \mathbb{T}_{2}, \sigma\right), h\right\rangle$ is contracting in the extended sense, i.e. there exist positive numbers $N$ and $v$ such that

$$
\rho\left(\pi\left(t, x_{1}\right), \pi\left(t, x_{2}\right)\right) \leq N \mathrm{e}^{-v t} \rho\left(x_{1}, x_{2}\right)
$$

for all $x_{1}, x_{2} \in X\left(h\left(x_{1}\right)=h\left(x_{2}\right)\right)$ and $t \in \mathbb{T}_{1}$;

(iv) $\Gamma(Y, X)=\{\gamma \mid \gamma:\} Y \rightarrow K(X)$ is a set-valued $\beta$-continuous mapping and $h(\gamma(y))=y$ for all $\{y \in Y\} \neq \varnothing$.

Then

(i) there exists a unique invariant section $\gamma \in \Gamma(Y, X)$ of the non-autonomous dynamical system $\left\langle\left(X, \mathbb{T}_{1}, \pi\right),\left(Y, \mathbb{T}_{2}, \sigma\right), h\right\rangle$;

(ii) the non-autonomous dynamical system $\left\langle\left(X, \mathbb{T}_{1}, \pi\right),\left(Y, \mathbb{T}_{2}, \sigma\right), h\right\rangle$ is compactly dissipative and its Levinson center $J=\gamma(Y)$;

(iii) $\bigcup\left\{\pi^{t} J_{q}: q \in\left(\sigma^{t}\right)^{-1}(y t)\right\}=J_{\sigma(t, y)}$ for all $t \in \mathbb{T}_{1}$ and $y \in Y$;

(iv) if $\left(Y, \mathbb{T}_{2}, \sigma\right)$ is a group dynamical system (i.e. $\mathbb{T}_{2}=\mathbb{S}$ ), then the unique invariant section $\gamma$ of the non-autonomous dynamical system $\left\langle\left(X, \mathbb{T}_{1}, \pi\right),\left(Y, \mathbb{T}_{2}, \sigma\right), h\right\rangle$ is onevalued (i.e. $\gamma(y)$ consists of a single point for any $y \in Y)$ and

$$
\rho(\pi(t, x), \pi(t, \gamma(h(x)))) \leq N \mathrm{e}^{-v t} \rho(x, \gamma(h(x)))
$$

for all $x \in X$ and $t \in \mathbb{T}$.

Proof. Since the space $Y$ is compact and invariant, then according to Theorem 2.1 the semigroup dynamical system $(Y, \mathbb{T}, \sigma)$ can be prolonged to a group set-valued dynamical system $(Y, \mathbb{S}, \tilde{\sigma})$ (this means that $\tilde{\sigma}(s, y)=\sigma(s, y)$ for all $(s, y) \in \mathbb{T} \times Y)$.

Let us denote by $\alpha: C(X) \times C(X) \rightarrow \mathbb{R}_{+}$the Hausdorff distance on $K(X)$ and by $d: \Gamma(Y, X) \times \Gamma(Y, X) \rightarrow \mathbb{R}_{+}$the function defined by the equality

$$
d\left(\gamma_{1}, \gamma_{2}\right):=\sup _{y \in Y} \alpha\left(\gamma_{1}(y), \gamma_{2}(y)\right) .
$$


It is easy to verify that by equality (5) there is defined a distance on $\Gamma(Y, X)$. We will show that the metric space $(\Gamma(Y, X), d)$ is complete. In fact, let $\left\{\gamma_{n}\right\} \subset \Gamma(Y, X)$ be a sequence satisfying the condition

$$
d\left(\gamma_{n}, \gamma_{m}\right) \rightarrow 0
$$

as $n, m \rightarrow+\infty$ and $y \in Y$. From (6) it follows that the sequence $\left\{\gamma_{n}(y)\right\} \subset K(X)$ is convergent in the space $(K(X), \alpha)$. We denote by $\gamma: Y \rightarrow K(X)$ the mapping defined by the equality

$$
\gamma(y):=\lim _{n \rightarrow+\infty} \gamma_{n}(y)
$$

(for any $y \in Y$ ). Let $\varepsilon>0$ be an arbitrary real number; then according to (6) there exists $n(\varepsilon) \in \mathbb{N}$ such that

$$
\alpha\left(\gamma_{n}(y), \gamma_{m}(y)\right) \leq \frac{\varepsilon}{4}
$$

for all $n, m \geq n(\varepsilon)$. Passing to the limit as $m \rightarrow+\infty$ and taking into consideration (7), we obtain that

$$
\alpha\left(\gamma_{n}(y), \gamma(y)\right) \leq \frac{\varepsilon}{4}
$$

for all $n \geq n(\varepsilon)$ and $y \in Y$.

Let now $y \in Y$ and $\left\{y_{k}\right\} \rightarrow y$; then

$$
\begin{aligned}
& \beta\left(\gamma\left(y_{k}\right), \gamma(y)\right) \leq \beta\left(\gamma\left(y_{k}\right), \gamma_{n(\varepsilon)}(y)\right)+\beta\left(\gamma_{n(\varepsilon)}\left(y_{k}\right), \gamma_{n(\varepsilon)}(y)\right) \\
& \quad+\beta\left(\gamma_{n(\varepsilon)(y)}, \gamma(y)\right) \leq 2 d\left(\gamma_{n(\varepsilon)}, \gamma\right)+\beta\left(\gamma_{n(\varepsilon)}\left(y_{k}\right), \gamma_{n(\varepsilon)}(y)\right) .
\end{aligned}
$$

From (9) and (10) it follows that

$$
\beta\left(\gamma\left(y_{k}\right), \gamma(y)\right) \leq \frac{\varepsilon}{2}+\beta\left(\gamma_{n(\varepsilon)}\left(y_{k}\right), \gamma_{n(\varepsilon)}(y)\right)
$$

and, consequently,

$$
\limsup _{k \rightarrow+\infty} \beta\left(\gamma\left(y_{k}\right), \gamma(y)\right) \leq \frac{\varepsilon}{2}+\limsup _{k \rightarrow+\infty} \beta\left(\gamma_{n(\varepsilon)}\left(y_{k}\right), \gamma_{n(\varepsilon)}(y)\right) \text {. }
$$

Since $\gamma_{n(\varepsilon)} \in \Gamma(Y, X)$, then

$$
\limsup _{k \rightarrow+\infty} \beta\left(\gamma_{n(\varepsilon)}\left(y_{k}\right), \gamma_{n(\varepsilon)}(y)\right)=\lim _{k \rightarrow+\infty} \beta\left(\gamma_{n(\varepsilon)}\left(y_{k}\right), \gamma_{n(\varepsilon)}(y)\right)=0 .
$$

From (12) and (13) we obtain

$$
\limsup _{k \rightarrow+\infty} \beta\left(\gamma\left(y_{k}\right), \gamma(y)\right) \leq \frac{\varepsilon}{2}<\varepsilon .
$$

As $\varepsilon$ is an arbitrary positive number, then from (14) it follows that

$$
\lim _{k \rightarrow+\infty} \beta\left(\gamma\left(y_{k}\right), \gamma(y)\right)=0,
$$

i.e. $\gamma \in \Gamma(Y, X)$. Finally from (9) we obtain that $d\left(\gamma_{n}, \gamma\right) \rightarrow 0$ as $n \rightarrow+\infty$.

Let $t \in \mathbb{T}_{1}$; by $S^{t}$ we denote the mapping of $\Gamma(Y, X)$ to itself defined by the equality $\left(S^{t} \gamma\right)(y)=\pi\left(t, \gamma\left(\left(\sigma^{t}\right)^{-1} y\right)\right)$ for all $t \in \mathbb{T}_{1}, y \in Y$ and $\gamma \in \Gamma(Y, X)$. It is easy to see that $S^{t} \gamma \in \Gamma(Y, X), S^{t} S^{\tau}=S^{t+\tau}$ for all $t, \tau \in \mathbb{T}_{1}$ and $\gamma \in \Gamma(Y, X)$ and, hence, $\left\{S^{t}\right\}_{t \in \mathbb{T}_{1}}$ forms 
a commutative semi-group. Besides this, from inequality (3) and the definition of the metric $d$, under the conditions of the theorem, the next inequality follows:

$$
d\left(S^{t} \gamma_{1}, S^{t} \gamma_{2}\right) \leq \mathcal{N} \mathrm{e}^{-v t} d\left(\gamma_{1}, \gamma_{2}\right)
$$

for all $t \in \mathbb{T}_{1}$ and $\gamma_{i} \in \Gamma(Y, X)(i=1,2)$. To prove inequality (15) it is sufficient to show that

$$
\alpha\left(\pi^{t} \gamma_{1}\left(\sigma^{-t} y\right)\right), \pi^{t} \gamma_{2}\left(\sigma^{-t} y\right) \leq \mathcal{N} \mathrm{e}^{-v t} d\left(\gamma_{1}, \gamma_{2}\right)
$$

for all $y \in Y$, where $\sigma^{-t} y:=\{q \in Y \mid \sigma(t, q)=y\}$.

Let $v \in \pi^{t} \gamma_{2}\left(\sigma^{-t} y\right)$ be an arbitrary element; then there is $q \in \sigma^{-t} y$ and $x_{2}(y) \in \gamma_{2}(q)$ such that $v=\pi^{t} x_{2}(y)$. We choose $x_{1}(y) \in \gamma_{1}(q)$ such that

$$
\rho\left(x_{1}(y), x_{2}(y)\right) \leq \alpha\left(\gamma_{1}(q), \gamma_{2}(q)\right) \leq d\left(\gamma_{1}, \gamma_{2}\right)
$$

(by compactness of $\gamma_{i}(q)(i=1,2)$, obviously there exists such an $x_{1}(y)$ and additionally $\left.h\left(x_{1}(y)\right)=h\left(x_{2}(y)\right)=q\right)$. Then we have

$$
\rho\left(\pi^{t} x_{1}(y), \pi^{t} x_{2}(y)\right) \leq \mathcal{N} \mathrm{e}^{-v t} \rho\left(x_{1}(y), x_{2}(y)\right) \leq \mathcal{N} \mathrm{e}^{-v t} d\left(\gamma_{1}, \gamma_{2}\right),
$$

i.e. for all $v \in \pi^{t} \gamma_{2}\left(\sigma^{-t} y\right)$ there exists $u:=\pi^{t} x_{1}(y) \in \pi^{t} \gamma_{1}\left(\sigma^{-t} y\right)$ such that $\rho(u, v) \leq \mathcal{N} \mathrm{e}^{-v t} d\left(\gamma_{1}, \gamma_{2}\right)$. This means that $\beta\left(\pi^{t} \gamma_{1}\left(\sigma^{-t} y\right), \pi^{t} \gamma_{2}\left(\sigma^{-t} y\right)\right) \leq \mathcal{N} \mathrm{e}^{-v t} d\left(\gamma_{1}, \gamma_{2}\right)$. Analogously, we can establish the inequality $\beta\left(\pi^{t} \gamma_{2}\left(\sigma^{-t} y\right), \pi^{t} \gamma_{1}\left(\sigma^{-t} y\right)\right) \leq \mathcal{N} \mathrm{e}^{-v t} d\left(\gamma_{1}, \gamma_{2}\right)$ and, consequently, $\alpha\left(\pi^{t} \gamma_{1}\left(\sigma^{-t} y\right), \pi_{\lambda}^{t} \gamma_{2}\left(\sigma^{-t} y\right)\right) \leq \mathcal{N} \mathrm{e}^{-v t} d\left(\gamma_{1}, \gamma_{2}\right)$ for all $y \in Y$ and $t \in \mathbb{T}_{1}$. Thus, we have

$$
d\left(S^{t} \gamma_{1}, S^{t} \gamma_{2}\right) \leq N \mathrm{e}^{-v t} d\left(\gamma_{1}, \gamma_{2}\right)
$$

for all $t \in \mathbb{T}_{1}$ and $\gamma_{1}, \gamma_{2} \in \Gamma(Y, X)$. From inequality (18) it follows that for $t$ large enough the mappings $S^{t}$ of the space $\Gamma(Y, X)$ are contractions, and since $\left\{S^{t}\right\}_{t \in \mathbb{T}_{1}}$ is commutative there exists a unique common stationary point $\gamma$ which is an invariant section of a non-autonomous dynamical system $\left\langle\left(X, \mathbb{T}_{1}, \pi\right),\left(Y, \mathbb{T}_{2}, \sigma\right), h\right\rangle$, i.e. $\gamma(Y) \subset X$ is an invariant set of the dynamical system $(X, \mathbb{T}, f)$.

Let us write $K:=\gamma(Y)$; then $K$ is a non-empty compact and invariant set of the dynamical system $\left(X, \mathbb{T}_{1}, \pi\right)$. From inequality (3) it follows that

$$
\lim _{t \rightarrow+\infty} \rho\left(\pi^{t} M, K\right)=0
$$

for all $M \in K(X)$ and, consequently, the dynamical system $\left(X, \mathbb{T}_{1}, \pi\right)$ is compactly dissipative and its Levinson center $J \subseteq K$. On the other hand, $K \subseteq J$, because the set $K=\gamma(Y)$ is compact and invariant, but $\bar{J}$ is a maximal compact invariant set of $\left(X, \mathbb{T}_{1}, \pi\right)$. Thus we have $J=\gamma(Y)$.

Let now $\mathbb{T}_{2}=\mathbb{S}$. Then we will show that the set $\gamma(y)$ contains a single point for any $y \in Y$. If we suppose that this is not true, then there are $y_{0} \in Y$ and $x_{1}, x_{2} \in \gamma\left(y_{0}\right)\left(x_{1} \neq x_{2}\right)$. Let $\phi_{i} \in \Phi_{x_{i}}$ $(i=1,2)$ be such that $\phi_{i}(\mathbb{S}) \subseteq J$. Then we have

$$
\pi^{t}\left(\phi_{i}(-t)\right)=x_{i} \quad(i=1,2)
$$

for all $t \in \mathbb{T}_{1}$. Note that from inequality (3) and equality (20) it follows that

$$
\rho\left(x_{1}, x_{2}\right)=\rho\left(\pi^{t}\left(\phi_{1}(-t)\right), \pi^{t}\left(\phi_{2}(-t)\right)\right) \leq N \mathrm{e}^{-v t} \rho\left(\phi_{1}(-t), \phi_{2}(-t)\right) \leq N \mathrm{e}^{-v t} C
$$

for all $t \in \mathbb{T}$, where $C:=\sup \left\{\rho\left(\phi_{1}(s), \phi_{2}(s)\right): s \in \mathbb{S}\right\}$. Passing to the limit in (21)) as $t \rightarrow+\infty$ we obtain $x_{1}=x_{2}$. The contradiction obtained proves our statement. 
Thus, if $\mathbb{T}_{2}=\mathbb{S}$, the unique fixed point $\gamma \in \Gamma(Y, X)$ of the semi-group of operators $\left\{S^{t}\right\}_{t \in \mathbb{T}_{1}}$ is a single-valued function and, consequently, it is continuous. Finally, inequality (4) follows from (3), because $h(\gamma(h(x)))=(h \circ \gamma)(h(x))=h(x)$ for all $x \in X$. The theorem is completely proved.

Remark 3.3. 1. Under the conditions of Theorem 3.2, if $\left(Y, \mathbb{T}_{2}, \sigma\right)$ is a group dynamical system (i.e. $\mathbb{T}_{2}=\mathbb{S}$ ), then the unique invariant section $\gamma$ of the non-autonomous dynamical system $\left\langle\left(X, \mathbb{T}_{1}, \pi\right),\left(Y, \mathbb{T}_{2}, \sigma\right), h\right\rangle$ is one-valued (i.e. $\gamma(y)$ consists of a single point for any $\left.y \in Y\right)$. Analogous statements for non-autonomous dynamical systems are known (see, for example, [12, Ch.2] and [33]). For the case of semi-group dynamical system $\left(Y, \mathbb{T}_{2}, \sigma\right)$ (i.e. where the mapping $\sigma(t, \cdot): Y \mapsto Y$ is not invertible) Theorem 3.2 is formulated and proved for the first time in this paper.

2. If $\left(Y, \mathbb{T}_{2}, \sigma\right)$ is a semi-group dynamical system (i.e. $\mathbb{T}_{2}=\mathbb{R}_{+}$or $\left.\mathbb{Z}_{+}\right)$, then the unique invariant section $\gamma$ of the non-autonomous dynamical system $\left\langle\left(X, \mathbb{T}_{1}, \pi\right),\left(Y, \mathbb{T}_{2}, \sigma\right), h\right\rangle$ is multivalued (i.e. $\gamma(y)$ contains, generally speaking, more than one point). This is confirmed by the example below, which is a slight modification of an example from [32, Ch.1,p.42-43].

Example 3.4. Let $Y:=[-1,1]$ and $\left(Y, \mathbb{Z}_{+}, \sigma\right)$ be a cascade generated by positive powers of the odd function $g$, defined on $[0,1]$ in the following way:

$$
g(y)= \begin{cases}-2 y, & 0 \leq y \leq \frac{1}{2} \\ 2(y-1), & \frac{1}{2}<y \leq 1\end{cases}
$$

It is easy to check that $g(Y)=Y$. Let us put $X:=\mathbb{R} \times Y$ and denote by $\left(X, \mathbb{Z}_{+}, \pi\right)$ a discrete dynamical system generated by the positive powers of the mapping $P: X \rightarrow X$ :

$$
P\left(\begin{array}{l}
u \\
y
\end{array}\right)=\left(\begin{array}{c}
f(u, y) \\
g(y)
\end{array}\right),
$$

where $f(u, y):=\frac{1}{10} u+\frac{1}{2} y$. Finally, let $h=p r_{2}: X \rightarrow Y$. From (22)), it follows that $h$ is a homomorphism of $\left(X, \mathbb{Z}_{+}, \pi\right)$ onto $\left(Y, \mathbb{Z}_{+}, \sigma\right)$ and, consequently, $\left\langle\left(X, \mathbb{Z}_{+}, \pi\right),\left(Y, \mathbb{Z}_{+}, \sigma\right), h\right\rangle$ is a non-autonomous dynamical system. Note that

$$
\left|\left(u_{1}, y\right)-\left(u_{2}, y\right)\right|=\left|u_{1}-u_{2}\right|=10\left|P\left(u_{1}, y\right)-P\left(u_{2}, y\right)\right| .
$$

From (23), it follows that

$$
\left|P^{n}\left(u_{1}, y\right)-P^{n}\left(u_{2}, y\right)\right| \leq \mathcal{N} \mathrm{e}^{-v n}\left|\left\langle u_{1}, y\right\rangle-\left\langle u_{2}, y\right\rangle\right|
$$

for all $n \in \mathbb{Z}_{+}$, where $\mathcal{N}=1$ and $v=\ln 10$. By Theorem 3.2 there exists a unique $\beta$-continuous invariant section $\gamma \in \Gamma(Y, X)$ of the non-autonomous dynamical system $\left\langle\left(X, \mathbb{Z}_{+}, \pi\right),\left(Y, \mathbb{Z}_{+}, \sigma\right), h\right\rangle$. According to Sharkovsky et al. [32, p. 43], $\gamma(y)$ is homeomorphic to the Cantor set for all $y \in[-1,1]$.

Remark 3.5. Let $Y$ be a topological space, $(W, \rho)$ be a metric space, $(X, h, Y)$ be a trivial bundle fiber (this means that $X:=W \times Y$ and $\left.h:=p r_{2}: X \rightarrow Y\right)$ and $(X, \mathbb{T}, \pi)$ be a skew-product dynamical system, i.e. $\pi:=(\varphi, \sigma) \pi(t,(u, y)):=(\varphi(t, u, y), \sigma(t, y))$. Then Theorem 3.2 is also true, although the space $X$ is in general not metrizable. 


\section{Discrete inclusions, the ensemble of dynamical systems (collages) and cocycles}

Let $W$ be a topological space. Denote by $C(W)$ the space of all continuous operators $f: W \rightarrow W$ equipped with the compact-open topology. Consider a set of operators $\mathcal{M} \subseteq C(W)$ and an ensemble (collage) of discrete dynamical systems $(W, f)_{f \in \mathcal{M}}$.

A discrete inclusion $D I(\mathcal{M})$ is the name given to (see, for example, $[22,6,18]$ ) a set of all sequences $\left\{\left\{x_{j}\right\} \mid j \geq 0\right\} \subset W$ such that

$$
x_{j}=f_{i_{j}} x_{j-1}
$$

for some $f_{i_{j}} \in \mathcal{M}$ (a trajectory of $\operatorname{DI}(\mathcal{M})$ ), i.e.

$$
x_{j}=f_{i_{j}} f_{i_{j-1}} \ldots f_{i_{1}} x_{0} \quad \text { all } f_{i_{k}} \in \mathcal{M} .
$$

A bilateral sequence is the name given to $\left\{\left\{x_{j}\right\} \mid j \in \mathbb{Z}\right\} \subset W$ a full trajectory of $\operatorname{DI}(\mathcal{M})$ (an entire trajectory or trajectory on $\mathbb{Z}$ ) if $x_{n+j}=f_{i_{j}} x_{n+j-1}$ for all $n \in \mathbb{Z}$.

Let us consider the set-valued function $F: W \rightarrow K(W)$ defined by the equality $F(x):=$ $\{f(x) \mid f \in \mathcal{M}\}$. Then the discrete inclusion $D I(\mathcal{M})$ is equivalent to the difference inclusion

$$
x_{j} \in F\left(x_{j-1}\right) .
$$

We denote by $\mathcal{F}_{x_{0}}$ the set of all trajectories of discrete inclusion (25) (or $D I(\mathcal{M})$ ) issuing from the point $x_{0} \in W$ and $\mathcal{F}:=\bigcup\left\{\mathcal{F}_{x_{0}} \mid x_{0} \in W\right\}$.

Below we will give a new approach as regards the study of discrete inclusions $D I(\mathcal{M})$ (or difference inclusion (25)). Denote by $C\left(\mathbb{Z}_{+}, W\right)$ the space of all continuous mappings $f: \mathbb{Z}_{+} \rightarrow W$ equipped with the compact-open topology. Denote by $\left(C\left(\mathbb{Z}_{+}, X\right), \mathbb{Z}_{+}, \sigma\right)$ a dynamical system of translations (shifts dynamical system or dynamical system of Bebutov [29, 30]) on $C\left(\mathbb{Z}_{+}, W\right)$, i.e. $\sigma(k, f):=f_{k}$ and $f_{k}$ is a $k \in \mathbb{Z}_{+}$shift of $f$ (i.e. $f_{k}(n):=f(n+k)$ for all $n \in \mathbb{Z}_{+}$).

We may now rewrite Eq. (25) in the following way:

$$
x_{j+1}=\omega(j) x_{j}, \quad\left(\omega \in \Omega:=C\left(\mathbb{Z}_{+}, \mathcal{M}\right)\right)
$$

where $\omega \in \Omega$ is the operator function defined by the equality $\omega(j):=f_{i_{j+1}}$ for all $j \in \mathbb{Z}_{+}$. We denote by $\varphi\left(n, x_{0}, \omega\right)$ the solution of Eq. (26) issuing from the point $x_{0} \in E$ at the initial moment $n=0$. Note that $\mathcal{F}_{x_{0}}=\left\{\varphi\left(\cdot, x_{0}, \omega\right) \mid \omega \in \Omega\right\}$ and $\mathcal{F}=\left\{\varphi\left(\cdot, x_{0}, \omega\right) \mid x_{0} \in W, \omega \in \Omega\right\}$, i.e. $D I(\mathcal{M})$ (or inclusion (25)) is equivalent to the family of non-autonomous Eq. (26) $(\omega \in \Omega)$.

From the general properties of difference equations it follows that the mapping $\varphi: \mathbb{Z}_{+} \times W \times$ $\Omega \rightarrow W$ satisfies the following conditions:

(i) $\varphi\left(0, x_{0}, \omega\right)=x_{0}$ for all $\left(x_{0}, \omega\right) \in W \times \Omega$;

(ii) $\varphi\left(n+\tau, x_{0}, \omega\right)=\varphi\left(n, \varphi\left(\tau, x_{0}, \omega\right), \sigma(\tau, \omega)\right)$ for all $n, \tau \in \mathbb{Z}_{+}$and $\left(x_{0}, \omega\right) \in W \times \Omega$;

(iii) the mapping $\varphi$ is continuous;

(iv) for any $n, \tau \in \mathbb{Z}_{+}$and $\omega_{1}, \omega_{2} \in \Omega$ there exists $\omega_{3} \in \Omega$ such that

$$
U\left(n, \omega_{2}\right) U\left(\tau, \omega_{1}\right)=U\left(n+\tau, \omega_{3}\right),
$$

where $\omega \in \Omega, U(n, \omega):=\varphi(n, \cdot, \omega)=\prod_{k=0}^{n} \omega(k), \omega(k):=f_{i_{k}}(k=0,1, \ldots, n)$ and $f_{i_{0}}:=I d_{W}$.

Let $W, \Omega$ be two topological spaces and $(\Omega, \mathbb{T}, \sigma)$ be a semi-group dynamical system on $\Omega$.

Recall [29] that a triplet $\langle W, \varphi,(\Omega, \mathbb{T}, \sigma)\rangle$ (or briefly $\varphi$ ) is called a cocycle over $(\Omega, \mathbb{T}, \sigma$ ) with the fiber $W$ if $\varphi$ is a mapping from $\mathbb{T} \times W \times \Omega$ to $W$ satisfying the following conditions: 
1. $\varphi(0, x, \omega)=x$ for all $(x, \omega) \in W \times \Omega$;

2. $\varphi(n+\tau, x, \omega)=\varphi(n, \varphi(\tau, x, \omega), \sigma(\tau, \omega))$ for all $n, \tau \in \mathbb{T}$ and $(x, \omega) \in W \times \Omega$;

3. the mapping $\varphi$ is continuous.

Let $X:=W \times \Omega$, and define the mapping $\pi: X \times \mathbb{T} \rightarrow X$ by the equality $\pi((u, \omega), t):=$ $(\varphi(t, u, \omega), \sigma(t, \omega))$ (i.e. $\pi=(\varphi, \sigma))$. Then it is easy to check that $(X, \mathbb{T}, \pi)$ is a dynamical system on $X$, which is called a skew-product dynamical system [1,29]; but $h=p r_{2}: X \rightarrow \Omega$ is a homomorphism of $(X, \mathbb{T}, \pi)$ onto $(\Omega, \mathbb{T}, \sigma)$ and hence $\langle(X, \mathbb{T}, \pi),(\Omega, \mathbb{T}, \sigma), h\rangle$ is a nonautonomous dynamical system.

Thus, if we have a cocycle $\langle W, \varphi,(\Omega, \mathbb{T}, \sigma)\rangle$ over the dynamical system $(\Omega, \mathbb{T}, \sigma)$ with the fiber $W$, then there can be constructed a non-autonomous dynamical system $\left\langle\left(X, \mathbb{T}_{1}, \pi\right),(\Omega, \mathbb{T}, \sigma), h\right\rangle(X:=W \times \Omega)$, which we will call a non-autonomous dynamical system generated by (associated with) the cocycle $\langle W, \varphi,(\Omega, \mathbb{T}, \sigma)\rangle$ over $(\Omega, \mathbb{T}, \sigma)$.

From the above it follows that every $D I(\mathcal{M})$ (respectively, inclusion (25)) in a natural way generates a cocycle $\left\langle W, \varphi,\left(\Omega, \mathbb{Z}_{+}, \sigma\right)\right\rangle$, where $\Omega=C\left(\mathbb{Z}_{+}, \mathcal{M}\right),\left(\Omega, \mathbb{Z}_{+}, \sigma\right)$ is a dynamical system of shifts on $\Omega$ and $\varphi(n, x, \omega)$ is the solution of Eq. (26) issuing from the point $x \in W$ at the initial moment $n=0$. Thus, we can study inclusion (25) (respectively, $D I(\mathcal{M})$ ) in the framework of the theory of cocycles with discrete time.

Theorem 4.1. Let $\mathcal{M}$ be a compact subset of $C(W)$ and $\left\langle W, \phi,\left(\Omega, \mathbb{Z}_{+}, \sigma\right)\right\rangle$ be a cocycle generated by $\mathrm{DI}(\mathcal{M})$. Then

(i) $\Omega=\overline{\operatorname{Per}(\sigma)}$, where $\operatorname{Per}(\sigma)$ is the set of all periodic points of $\left(\Omega, \mathbb{Z}_{+}, \sigma\right)$ (i.e. $\omega \in \operatorname{Per}(\sigma)$ if there exists $\tau \in \mathbb{N}$ such that $\sigma(\tau, \omega)=\omega)$;

(ii) the set $\Omega$ is compact;

(iii) $\Omega$ is invariant, i.e. $\sigma^{t} \Omega=\Omega$ for all $t \in \mathbb{Z}_{+}$;

(iv) $\varphi$ satisfies the condition (27).

Proof. Let $Y=\Omega:=C\left(\mathbb{Z}_{+}, Q\right)$ and $\left(Y, \mathbb{Z}_{+}, \sigma\right)$ be a semi-group dynamical system of shifts on $Y$. According to the theorem of Tikhonoff (see, for example, [24]), $\Omega$ is compact. We denote by $\operatorname{Per}(\sigma)$ the set of all periodic points of the dynamical system $\left(\Omega, \mathbb{Z}_{+}, \sigma\right)$, i.e. $\operatorname{Per}(\sigma):=\{\omega \in$ $\Omega: \exists \tau \in \mathcal{N}$ such that $\sigma(\tau, \omega)=\omega\}$. We will prove that $\overline{\operatorname{Per}(\sigma)}=\Omega$, i.e. the set of all periodic points of $\Omega$ is dense in $\Omega$. In fact, if $\omega \in \Omega$, then denote by $\omega_{k}$ the periodic point from $\operatorname{Per}(\sigma)$ such that $\omega_{k}(t):=\omega(t)$ for all $t=0,1, \ldots, k-1$. It is easy to see that $\left\{\omega_{k}\right\} \rightarrow \omega$ in $\Omega$ (the convergence in $\Omega$ is the convergence on compacts from $\mathbb{Z}_{+}$). From the fact established above it follows that $\Omega$ is invariant, i.e. $\sigma^{t} \Omega=\Omega$. In fact, let $\omega \in \Omega, t \in \mathbb{Z}_{+}$and $\left\{\omega_{k}\right\} \subset \operatorname{Per}(\sigma)$ be such that $\left\{\omega_{k}\right\} \rightarrow \omega$. Let $\tau_{k} \in \mathbb{N}$ be such that $\sigma\left(\tau_{k}, \omega_{k}\right)=\omega_{k}$ and $\tau_{k} \rightarrow+\infty$. Then there exists $k_{0}=k_{0}(t)$ such that $\tau_{k} \geq t$ for all $k \geq k_{0}$ and, consequently, we have

$$
\omega_{k}=\sigma\left(\tau_{k}, \omega_{k}\right)=\sigma\left(t, \sigma\left(\tau_{k}-t, \omega_{k}\right)\right)
$$

for all $k \geq k_{0}$. Since the space $\Omega$ is compact we may suppose that the sequence $\left\{\sigma\left(\tau_{k}-t, \omega_{k}\right)\right\}$ is convergent. Let $\bar{\omega}:=\lim _{k \rightarrow+\infty} \sigma\left(\tau_{k}-t, \omega_{k}\right)$; then from equality (28) we obtain $\omega=\sigma(t, \bar{\omega})$, i.e. $\sigma^{t} \Omega=\Omega$.

Remark 4.2. The first statement of Theorem 4.1 is a slight generalization of a well-known result (see, for example, [35, Ch.III]).

Let $(W, \rho)$ be a metric space. 
A mapping $f: W \rightarrow W$ satisfies the condition of Lipschitz if there exists a constant $L>0$ such that $\rho\left(f\left(x_{1}\right), f\left(x_{2}\right)\right) \leq L \rho\left(x_{1}, x_{2}\right)$ for all $x_{1}, x_{2} \in W$. The smallest constant with the above-mentioned property is called the constant of Lipschitz $L(f)$ of the mapping $f$.

A subset of operators $\mathcal{M} \subseteq C(W)$ is said to be generally contracting (contracting in the extended sense) if there are positive numbers $\mathcal{N}$ and $\alpha<1$ such that

$$
L\left(f_{i_{n}} \circ f_{i_{n-1}} \circ \cdots \circ f_{i_{1}}\right) \leq \mathcal{N} \alpha^{n}
$$

for all $f_{i_{1}}, f_{i_{2}}, \ldots, f_{i_{n}} \in \mathcal{M}$ and $n \in \mathbb{N}$.

Example 4.3. Let $W:=C[0,1]$, and $f \in C(W)$ be defined by the equality

$$
(f \varphi)(t):=\frac{3}{2} \int_{0}^{t} \varphi(s) \mathrm{d} s
$$

$(t \in[0,1]$ and $\varphi \in C[0,1])$. It is easy to see that $L\left(f^{n}\right)=\left(\frac{3}{2}\right)^{n} \frac{1}{n !}$, where $f^{n}:=f^{n-1} \circ f$ $(n=2,3, \ldots)$. In particular, $L(f)=\frac{3}{2}, L\left(f^{2}\right)=\frac{9}{8}$ and $L\left(f^{3}\right)=\frac{27}{32}<1$. Additionally, $L\left(f^{n}\right) \leq 2\left(\frac{3}{4}\right)^{n}$ for all $n \in \mathbb{N}$. Thus, the set $\mathcal{M}=\{f\}$ is generally contracting.

Theorem 4.4. Suppose that the following conditions are fulfilled:

(i) $\mathcal{M}$ is a compact subset of $C(W)$;

(ii) $\mathcal{M}$ is contracting in the extended sense.

Then:

(i) $I_{\omega}:=\{u \in W:$ a solution $\varphi(n, u, \omega)$ of $E q$. (26) is defined on $\mathbb{Z}$ and $\varphi(\mathbb{Z}, u, \omega)$ is relatively compact $\} \neq \emptyset$ for all $\omega \in \Omega$, i.e. every $E q$. (26) admits at least one solution defined on $\mathbb{Z}$ with a relatively compact range of values;

(ii) the sets $I_{\omega}(\omega \in \Omega)$ and $I:=\bigcup\left\{I_{\omega}: \omega \in \Omega\right\}$ are compact;

(iii) the set-valued map $\omega \rightarrow I_{\omega}$ is upper semi-continuous;

(iv) the family of compacts $\left\{I_{\omega}: \omega \in \Omega\right\}$ is invariant with respect to the cocycle $\varphi$, i.e.

$$
\bigcup\left\{\varphi\left(n, I_{q}, q\right): q \in\left(\sigma^{n}\right)^{-1}\left(\sigma^{n} \omega\right)\right\}=I_{\sigma^{n} \omega}
$$

for all $n \in \mathbb{Z}_{+}$and $\omega \in \Omega$;

(v) $\rho\left(\varphi\left(n, u_{1}, \omega\right), \varphi\left(n, u_{2}, \omega\right)\right) \leq \mathcal{N} \mathrm{e}^{-v n} \rho\left(u_{1}, u_{2}\right)$ for all $n \in \mathbb{Z}_{+}$and $\omega \in \Omega$ and $u_{1}, u_{2} \in W$, where $\mathcal{N}$ and $v$ are positive numbers from the definition of the contractivity of $\mathcal{M}$ in the extended sense;

(vi) if every map $f \in \mathcal{M}$ is invertible, then:

(a) $I_{\omega}$ consists of a single point $u_{\omega}$;

(b) the map $\omega \rightarrow u_{\omega}$ is continuous;

(c) $\varphi\left(t, u_{\omega}, \omega\right)=u_{\sigma(n, \omega)}$ for all $n \in \mathbb{Z}_{+}$and $\omega \in \Omega$;

(d) $\rho\left(\varphi(n, u, \omega), \varphi\left(n, u_{\omega}, \omega\right)\right) \leq \mathcal{N} \mathrm{e}^{-v n} \rho\left(u, u_{\omega}\right)$ for all $n \in \mathbb{Z}_{+}$and $\omega \in \Omega$.

Proof. Let $Y=\Omega:=C\left(\mathbb{Z}_{+}, Q\right)$ and $\left(Y, \mathbb{Z}_{+}, \sigma\right)$ be a semi-group dynamical system of shifts on $Y$. Then $Y$ is compact. By Theorem 4.4, $\overline{\operatorname{Per}(\sigma)}=\Omega$ and $\Omega$ is compact and invariant.

Let $\left\langle W, \varphi,\left(\Omega, \mathbb{Z}_{+}, \sigma\right)\right\rangle$ be a cocycle generated by $D I(\mathcal{M})$ (i.e. $\varphi(n, u, \omega):=U(n, \omega) u$, where $\left.U(n, \omega)=\prod_{k=0}^{n} \omega(k)(\omega \in \Omega)\right),\left(X, \mathbb{Z}_{+}, \pi\right)$ be a skew-product system associated with the 
cocycle $\varphi$ (i.e. $X:=W \times \Omega$ and $\pi:=(\varphi, \sigma))$ and $\left\langle\left(X, \mathbb{Z}_{+}, \pi\right),\left(Y, \mathbb{Z}_{+}, \sigma\right), h\right\rangle\left(h:=p r_{2}: X \rightarrow\right.$ $Y)$ be a non-autonomous dynamical system generated by the cocycle $\varphi$. Under the conditions of Theorem 4.4 we have

$$
\rho\left(\varphi\left(n, u_{1}, \omega\right), \varphi\left(n, u_{2}, \omega\right)\right) \leq \mathcal{N} \mathrm{e}^{-v n} \rho\left(u_{1}, u_{2}\right)
$$

for all $n \in \mathbb{Z}_{+}, u_{1}, u_{2} \in W$ and $\omega \in \Omega$, where $\nu:=-\ln \alpha(\mathcal{N}$ and $\alpha$ are positive numbers from the definition of the contraction of $\mathcal{M}$ in the extended sense). Now to finish the proof of the theorem it is sufficient to apply Theorem 3.2 (see also Remark 3.5) to the non-autonomous dynamical system $\left\langle\left(X, \mathbb{Z}_{+}, \pi\right),\left(\Omega, \mathbb{Z}_{+}, \sigma\right), h\right\rangle$ and write $I_{\omega}:=p r_{1}\left(J_{\omega}\right)$, where $J$ is the Levinson center of the dynamical system $\left(X, \mathbb{Z}_{+}, \pi\right), J_{\omega}:=J \bigcap h^{-1}(\omega)$ and $h:=p r_{2}$.

\section{Relation between compact global attractors of skew-product systems, collages and cocycles}

Let $(W, \rho)$ be a complete metric space and $\mathcal{M} \subseteq C(W)$ be a compact. Denote by $F$ the set-valued mapping defined by the equality

$$
F(u):=\{f(u): f \in \mathcal{M}, u \in W\} .
$$

Lemma 5.1. The following statements hold:

(i) the set $F(u)$ is a compact subset of $W$;

(ii) the set-valued mapping $F: W \rightarrow K(W)$ is upper semi-continuous.

Proof. Consider the mapping $G: W \times C(W) \rightarrow W$ defined by the equality $G(u, f):=f(u)$. Since $G$ is continuous (see, for example, [24, Ch.7]) and $\mathcal{M}$ is a compact subset of $C(W)$, then $F(u)=G(u, \mathcal{M})$ is compact too.

Let now $\left\{u_{n}\right\} \rightarrow u\left(u_{n}, u \in W\right), v_{n} \in F\left(u_{n}\right)$ and $\left\{v_{n}\right\} \rightarrow v$. We will show that $v \in F(u)$. In fact, since $v_{n} \in F\left(u_{n}\right)$, then there exists $f_{n} \in \mathcal{M}$ such that $v_{n}=f_{n}\left(u_{n}\right)$. We can suppose that the sequence $\left\{f_{n}\right\}$ is convergent in $C(W)$, because the set $\mathcal{M}$ is compact. Let $\left\{f_{n}\right\} \rightarrow f$. Then

$$
v:=\lim _{n \rightarrow+\infty} v_{n}=\lim _{n \rightarrow+\infty} f_{n}\left(v_{n}\right)=\lim _{n \rightarrow+\infty} G\left(u_{n}, f_{n}\right)=G(u, f)=f(u) \in F(u) .
$$

Corollary 5.2. Let $M \in K(W)$. Then $F(M) \in K(W)(F(M):=\{F(u): u \in M\})$.

Theorem $5.3([11])$. Let $(X, \mathbb{T}, \pi)$ be a set-valued dynamical system and let there be positive numbers $\mathcal{N}$ and $v$ such that

$$
\alpha\left(\pi\left(t, x_{1}\right), \pi\left(t, x_{2}\right)\right) \leq \mathcal{N} \mathrm{e}^{-v t} \rho\left(x_{1}, x_{2}\right)
$$

for all $x_{1}, x_{2} \in X$ and $t \in \mathbb{T}$. Then $(X, \mathbb{T}, \pi)$ is compactly dissipative.

Theorem 5.4. Suppose the following conditions are fulfilled:

(i) $\mathcal{M}:=\left\{f_{i}: i \in I\right\}$ is a compact subset from $C(W)$;

(ii) the set $\mathcal{M}$ of operators is contracting in the extended sense.

Then the set-valued discrete dynamical system $(W, F)$ is compactly dissipative. 
Proof. It is easy to verify that

$$
F^{n}=\left\{f_{i_{n}} \circ f_{i_{n-1}} \circ \cdots \circ f_{i_{1}}: i_{k} \in I(k=1,2, \ldots, n)\right\}=\{U(n, \omega): \omega \in \Omega\},
$$

where $\Omega:=C\left(\mathbb{Z}_{+}, \mathcal{M}\right), U(n, \omega):=\prod_{k=0}^{n} f_{i_{k}}=f_{i_{n}} \circ f_{i_{n-1}} \circ \cdots \circ f_{i_{1}} \circ f_{i_{o}}, \omega(k):=f_{i_{k}}$ $(k=1,2, \ldots, n)$ and $f_{i_{0}}:=I d_{W}$.

Let $\mathcal{N}>0$ and $v>0$ be constants from the definition of the extended contraction of the family of operators $\mathcal{M}$. We will prove that

$$
\alpha\left(F^{n}(A), F^{n}(B)\right) \leq \mathcal{N} \mathrm{e}^{-v n} \alpha(A, B)
$$

for all $A, B \in K(W)$. Indeed, let $v \in F^{n}(B)$. Since $F^{n}(B)=U(n, \Omega)(B)$, there exist $x_{2} \in B$ and $\omega \in \Omega$ such that $v=U(n, \omega) x_{2}$. We choose a point $x_{1} \in A$ such that $\rho\left(x_{1}, x_{2}\right) \leq \alpha(A, B)$. Then we have

$$
\begin{aligned}
\rho\left(U(n, \omega) x_{1}, v\right) & =\rho\left(U(n, \omega) x_{1}, U(n, \omega) x_{2}\right) \\
& \leq \mathcal{N} \mathrm{e}^{-v n} \rho\left(x_{2}, x_{1}\right) \leq \mathcal{N} \mathrm{e}^{-v n} \alpha(A, B) .
\end{aligned}
$$

Thus, for an arbitrary point $v \in F^{n}(B)$ there is a point $u:=U(n, \omega) x_{1} \in F^{n}(A)$ such that

$$
\rho(u, v) \leq \mathcal{N} \mathrm{e}^{-v n} \alpha(A, B)
$$

and, hence,

$$
\beta\left(F^{n}(A), F^{n}(B)\right) \leq \mathcal{N} \mathrm{e}^{-v n} \alpha(A, B) .
$$

Similarly we have the inequality

$$
\beta\left(F^{n}(B), F^{n}(A)\right) \leq \mathcal{N} \mathrm{e}^{-v n} \alpha(A, B) .
$$

Inequality (30) follows from inequalities (31) and (32). Now to finish the proof it is enough to cite Theorem 5.3.

Theorem 5.5. Let $\langle W, \phi,(\Omega, \mathbb{T}, \sigma)\rangle$ be a cocycle, $\Omega$ be compact and $f: \mathbb{T} \times W: \rightarrow K(W)$ be a mapping defined by the equality

$$
f(t, u)=\phi(t, u, \Omega)
$$

for all $u \in W$ and $t \in \mathbb{T}$.

Then the mapping $f$ possesses the following properties:

a. $f(0, u)=u$ for all $u \in W$;

b. $f(t, f(\tau, u)) \subseteq f(t+\tau, u)$ for all $t, \tau \in \mathbb{T}$ and $u \in W$;

c. $f: \mathbb{T} \times W \rightarrow K(W)$ is upper semi-continuous, i.e.

$$
\lim _{t \rightarrow t_{0}, u \rightarrow u_{0}} \beta\left(f(t, u), f\left(t_{0}, u_{0}\right)\right)=0 \quad \forall\left(t_{0}, u_{0}\right) \in \mathbb{T} \times W ;
$$

d. if the cocycle $\langle W, \phi,(\Omega, \mathbb{T}, \sigma)\rangle$ satisfies the following condition:

$$
\forall t, \tau \in \mathbb{T}, u_{1}, u_{2} \in W \exists u_{3} \text { suchthat } \phi\left(t, \phi\left(\tau, x, u_{1}\right), u_{2}\right)=\phi\left(t+\tau, x, u_{3}\right),
$$

then

$$
f(t, f(\tau, u))=f(t+\tau, u)
$$

for all $t, \tau \in \mathbb{T}$ and $u \in W$.

Proof. This statement follows directly from the corresponding definitions. 
Corollary 5.6. Every cocycle $\langle W, \phi,(\Omega, \mathbb{T}, \sigma)\rangle$ with the compact $\Omega$ and satisfying the condition (34) generates a set-valued dynamical system $(W, \mathbb{T}, f)$, where $f: \mathbb{T} \times W \rightarrow K(W)$ is defined by equality (33).

A cocycle $\varphi$ over $(\Omega, \mathbb{T}, \sigma)$ with the fiber $W$ is said to be a compactly dissipative one if there is a non-empty compact $K \subseteq W$ such that

$$
\lim _{t \rightarrow+\infty} \sup \{\beta(U(t, \omega) M, K) \mid \omega \in \Omega\}=0
$$

for any $M \in K(W)$, where $U(t, \omega):=\varphi(t, \cdot, \omega)$.

The smallest compact set $I \subseteq W$ with property (35) is said to be a Levinson center of the cocycle $\varphi$.

Theorem 5.7. The following affirmations hold:

(i) Let $\langle W, \phi,(\Omega, \mathbb{T}, \sigma)\rangle$ be a cocycle with the compact $\Omega$ and satisfying the condition (34). Then the following statements are equivalent:

(a) the cocycle $\varphi$ is compactly dissipative;

(b) the skew-product dynamical system $(X, \mathbb{T}, \pi)$ generated by the cocycle $\varphi$ is compactly dissipative;

(c) the set-valued dynamical system $(W, \mathbb{T}, f)$ generated by the cocycle $\varphi$ is compactly dissipative.

(ii) Let $\langle W, \phi,(\Omega, \mathbb{T}, \sigma)\rangle$ be a compact dissipative cocycle and the following conditions be fulfilled:

(a) $\Omega$ is compact and invariant $\left(\sigma^{t} \Omega=\Omega\right.$ for all $\left.t \in \mathbb{T}\right)$;

(b) the cocycle $\varphi$ satisfies condition (34).

Then $I=\operatorname{pr}_{1}(J)$, where $J$ is the Levinson center of the skew-product dynamical system $(X, \mathbb{T}, \pi)$ (generated by the cocycle $\varphi$ ) and $I$ is the Levinson center of the set-valued dynamical system $(W, \mathbb{T}, f)$ (generated by the cocycle $\varphi$ ).

Proof. Let $\langle W, \phi,(\Omega, \mathbb{T}, \sigma)\rangle$ be a cocycle with the compact $\Omega$ and $(X, \mathbb{T}, \pi)$ (respectively, $(W, \mathbb{T}, f))$ be the skew-product dynamical system (respectively, set-valued dynamical system) generated by the cocycle $\varphi$. If the cocycle $\varphi$ is compactly dissipative, then the skew-product dynamical system $(X, \mathbb{T}, \pi)$ (respectively, the set-valued dynamical system $(W, \mathbb{T}, f)$ ) will also be. In fact, if $K \in K(W)$ with property (35), then we have

$$
\lim _{t \rightarrow+\infty} \beta\left(\pi^{t} \tilde{M}, \tilde{K}\right)=0
$$

for any $\tilde{M} \in K(X)$, where $\tilde{K}:=K \times \Omega$. If we suppose that (36) is not true, then there exist $\varepsilon>0, \tilde{M}_{0} \in K(X),\left\{x_{n}\right\} \subseteq \tilde{M}_{0}$ and $\left\{t_{n}\right\} \rightarrow+\infty\left(t_{n} \in \mathbb{T}\right)$ such that

$$
\rho\left(\pi_{n}^{t} x_{n}, \tilde{K}\right) \geq \varepsilon_{0} .
$$

Let $x_{n}=\left(u_{n}, \omega_{n}\right)$. Since the set $\tilde{M}_{0}$, then the set $M_{0}:=\operatorname{pr}_{1}\left(\tilde{M}_{0}\right)\left(p r_{1}: X \rightarrow W\right)$ is compact too. According to the compact dissipativity of the cocycle $\varphi$ we can suppose that the sequence $\left(\left\{\varphi\left(t_{n}, u_{n}, \omega_{n}\right)\right\}\right)$ is convergent. By the compactness of $\Omega$ we can suppose that the sequence $\left\{\omega_{n}\right\} \subseteq \Omega$ is convergent too. Write $\bar{u}:=\lim _{n \rightarrow+\infty} \varphi\left(t_{n}, u_{n}, \omega_{n}\right)$ and $\bar{\omega}:=\lim _{n \rightarrow+\infty} \omega_{n}$. Then from (37) we obtain

$$
\rho(\bar{x}, \tilde{K}) \geq \varepsilon_{0},
$$


where $\bar{x}:=(\bar{u}, \bar{\omega})$. On the other hand, from equality (35) we have

$$
\rho(\bar{u}, K)=0
$$

and, hence, $\bar{x}=(\bar{u}, \bar{\omega}) \in K \times \Omega=\tilde{K}$. The last inclusion and (38) are contradictory. The contradiction obtained proves our statement.

Now we will prove that from the compact dissipativity of the skew-product dynamical system $(X, \mathbb{T}, \pi)$ there follows the compact dissipativity of the cocycle $\varphi$, which generates $(X, \mathbb{T}, \pi)$. In fact, let $(X, \mathbb{T}, \pi)$ be compactly dissipative and $J$ be its Levinson center. Write $I:=p r_{1}(J)$. It is clear that the set $I$ is non-empty and compact. We will show that

$$
\lim _{t \rightarrow+\infty} \sup \{\beta(U(t, \omega) M, I) \mid \omega \in \Omega\}=0
$$

for any $M \in K(W)$. If we suppose that (40) is not true, then there exist $\varepsilon_{0}>0,\left\{t_{n}\right\} \rightarrow+\in$ $\infty\left(t_{n} \in \mathbb{T}\right), M_{0} \in K(W),\left\{u_{n}\right\} \subseteq M_{0}$ and $\left\{\omega_{n}\right\} \subseteq \Omega$ such that

$$
\rho\left(\varphi\left(t_{n}, u_{n}, \omega_{n}\right), I\right) \geq \varepsilon_{0} .
$$

Let us write $\tilde{M}_{0}=M_{0} \times \Omega \in K(X)$. Since the skew-product dynamical system $(X, \mathbb{T}, \pi)$ is compactly dissipative and $J$ is its Levinson center, we have

$$
\lim _{t \rightarrow+\infty} \beta\left(\pi^{t} \tilde{M}_{0}, J\right)=0
$$

and, consequently,

$$
\lim _{t \rightarrow+\infty} \rho\left(\pi^{t} x_{n}, J\right)=0
$$

where $x_{n}:=\left(u_{n}, \omega_{n}\right)$ and $\pi^{t_{n}} x_{n}=\left(\varphi\left(t_{n}, u_{n}, \omega_{n}\right), \sigma\left(t_{n}, \omega_{n}\right)\right)$. By (42) we can suppose that the sequence $\left\{\pi^{t_{n}} x_{n}\right\}=\left\{\left(\varphi\left(t_{n}, u_{n}, \omega_{n}\right), \sigma\left(t_{n}, \omega_{n}\right)\right)\right\}$ is convergent and that its limit belongs to $J$. Let $\bar{x}:=\lim _{n \rightarrow+\infty} x_{n}=\left(\lim _{n \rightarrow+\infty} \varphi\left(t_{n}, u_{n}, \omega_{n}\right), \lim _{n \rightarrow+\infty} \sigma\left(t_{n}, \omega_{n}\right)\right):=(\bar{u}, \bar{\omega})$. Then $(\bar{u}, \bar{\omega}) \in J$ and $\bar{u} \in I_{\bar{\omega}} \subseteq I$, where $I_{\bar{\omega}}:=\operatorname{pr}_{1}\left(J_{\bar{\omega}}\right)$ and $J_{\bar{\omega}}:=J \bigcap\left(p r_{2}\right)^{-1}(\bar{\omega})$. Passing to the limit in inequality (41)) as $n \rightarrow+\infty$, we obtain that $\bar{u} \notin I$. The contradiction obtained proves our affirmation. Note that the set $I=p r_{1}(J)$ is the least compact subset of $W$ satisfying the condition (40). In fact, suppose that $I^{\prime}$ is a compact subset of $W$ possessing the property (40). We have

$$
\bigcup\left\{\varphi\left(t, I_{q}, \omega\right): q \in\left(\sigma^{t}\right)^{-1}(\sigma(t, \omega))\right\}=I_{\sigma(t, \omega)},
$$

since the sets $J$ and $\Omega$ are invariant. From equality (43) it follows that

$$
\begin{aligned}
& I=\bigcup\left\{I_{\sigma(t, \omega)}: \omega \in \Omega\right\} \subseteq \\
& \bigcup\left\{\varphi\left(t, I_{q}, \omega\right): q \in\left(\sigma^{t}\right)^{-1}(\sigma(t, \omega)), \omega \in \Omega\right\} \subseteq \\
& \varphi(t, I, \Omega)=f(t, I)
\end{aligned}
$$

for any $t \in \mathbb{T}$ and, hence, $I \subseteq I^{\prime}$. Finally we note that under the condition of the first part of the theorem the equivalence of conditions $\mathrm{b}$. and $\mathrm{c}$. follows from the equality $f(t, u)=\varphi(t, u, \Omega)$.

Let now $(X, \mathbb{T}, \pi)$ (respectively, $(W, \mathbb{T}, f)$ ) be the skew-product (respectively, set-valued) dynamical system generated by the cocycle $\varphi$ and $J$ (respectively, $I$ ) be its Levinson center. We denote by $I^{\prime}:=p r_{1}(J)$ and note that from the reasoning above we have

$$
\sup _{\omega \in \Omega} \beta\left(U(t, \omega) M, I^{\prime}\right)=\beta\left(U(t, \Omega) M, I^{\prime}\right)=\beta\left(f(t, M), I^{\prime}\right)=0
$$


for all $M \in K(W)$ and, consequently, $I \subseteq I^{\prime}$, as the set $I$ is the least compact subset of $W$ with property (44). On the other hand, from (44) we have $I \subseteq \varphi(t, I, \Omega)=f(t, I)$ for any $t \in \mathbb{T}$ and hence $I \subseteq \omega_{f}(I) \subseteq I^{\prime}$, where $\omega(I):=\bigcap_{t \geq 0} \overline{\bigcup_{\tau \geq t} f(\tau, I)}$. Thus, we obtain $I^{\prime}=I$. The theorem is completely proved.

Denote by $\Phi(\varphi)$ the set of all full trajectories of the cocycle $\varphi$.

Corollary 5.8. Let $\langle W, \phi,(\Omega, \mathbb{T}, \sigma)\rangle$ be a compactly dissipative cocycle and the following conditions be fulfilled:

(i) $\Omega$ is compact and invariant;

(ii) the cocycle $\varphi$ satisfies condition (34).

Then $I=\{u \in W: \exists \eta \in \Phi(\varphi), \eta(0)=u$ and $\eta(\mathbb{S})$ is relatively compact $\}$.

Proof. This statement follows from the equality $I=p r_{1}(J)$ and the invariance of the set $J$, because $\eta:=p r_{1}(\gamma) \in \Phi(\varphi)$ if $\gamma \in \Phi(\pi)$.

\section{Chaotic attractors of discrete control systems}

Theorem 6.1. Let $\mathcal{M}$ be a compact subset of $C(W)$. Suppose that the following conditions are fulfilled:

(i) $\mathcal{M}$ is a compact subset of $C(W)$;

(ii) $\mathcal{M}$ is contracting in the extended sense.

Then the following statements hold:

(i) the cocycle $\left\langle W, \varphi,\left(\Omega, \mathbb{Z}_{+}, \sigma\right)\right\rangle\left(\Omega:=C\left(\mathbb{Z}_{+}, \mathcal{M}\right)\right)$ is compactly dissipative;

(ii) if every map $f \in \mathcal{M}$ is invertible, then $I=\overline{\operatorname{Per}(\varphi)}$, where $\operatorname{Per}(\varphi):=\{u \in W: \exists \tau \in$ $\mathbb{N}$ and $\omega \in \Omega$ such that $\sigma(\tau, \omega)=\omega$ and $\varphi(\tau, u, \omega)=u\}$, where $I$ is the Levinson center of the cocycle $\varphi$.

Proof. Let $Y=\Omega:=C\left(\mathbb{Z}_{+}, Q\right)$ and $\left(Y, \mathbb{Z}_{+}, \sigma\right)$ be a semi-group dynamical system of shifts on $Y$. According to the theorem of Tikhonoff (see, for example, [24]), $Y$ is compact. Let us denote by $\operatorname{Per}(\sigma):=\{\omega \in \Omega: \exists \tau \in \mathcal{N}$ such that $\sigma(\tau, \omega)=\omega\}$ the set of all periodic points of the dynamical system $\left(\Omega, \mathbb{Z}_{+}, \sigma\right)$. Then $\overline{\operatorname{Per}(\sigma)}=\Omega$, i.e. the set of all periodic points of $\Omega$ is dense in $\Omega$ and $\Omega$ is invariant (see the proof of Theorem 4.4).

Let $\left\langle W, \varphi,\left(\Omega, \mathbb{Z}_{+}, \sigma\right)\right\rangle$ be a cocycle generated by $D I(\mathcal{M})$ (i.e. $\varphi(n, u, \omega):=U(n, \omega) u$, where $\left.U(n, \omega)=\prod_{k=o}^{n} \omega(k)(\omega \in \Omega)\right),\left(X, \mathbb{Z}_{+}, \pi\right)$ be a skew-product system associated with the cocycle $\varphi$ and $\left\langle\left(X, \mathbb{Z}_{+}, \pi\right),\left(Y, \mathbb{Z}_{+}, \sigma\right), h\right\rangle\left(h:=p r_{2}: X \rightarrow Y\right)$ be a non-autonomous dynamical system generated by the cocycle $\varphi$. By Theorem $4.4,\left(X, \mathbb{Z}_{+}, \pi\right)$ is compactly dissipative and its Levinson center $J$ is topologically and dynamically isomorphic to $\left(\Omega, \mathbb{Z}_{+}, \sigma\right)$. According to Theorem 5.7, $I=\operatorname{pr}_{1}(J)$. Since $\overline{\operatorname{Per}(\sigma)}=\Omega$, then $\overline{\operatorname{Per}(\pi)}=J$ and, consequently, $\overline{\operatorname{Per}(\varphi)}=I$.

The set $S \subset W$ is

(i) nowhere dense, provided that the interior of the closure of $S$ is the empty set, $\operatorname{int}(\operatorname{cl}(S))=\emptyset$;

(ii) totally disconnected, provided that the connected components are single points;

(iii) perfect, provided that it is closed and every point $p \in S$ is the limit of points $q_{n} \in S$ with $q_{n} \neq p$. 
The set $S \subset W$ is called a Cantor set, provided that it is totally disconnected, perfect and compact.

The subset $M$ of $(X, \mathbb{T}, \pi)$ is called (see, for example, [28]) chaotic if the following conditions hold:

(i) the set $M$ is transitive, i.e. there exists a point $x_{0} \in X$ such that $M=H\left(x_{0}\right):=$ $\overline{\left\{\pi\left(t, x_{0}\right): t \in \mathbb{T}\right\}}$

(ii) $M=\overline{\operatorname{Per}(\pi)}$, where $\operatorname{Per}(\pi)$ is the set of all periodic points of $(X, \mathbb{T}, \pi)$.

Recall that a point $x \in X$ of the dynamical system $(X, \mathbb{T}, \pi)$ is called Poisson stable in the positive direction if $x$ belongs to its $\omega$-limit set $\omega_{x}:=\bigcap_{t \geq 0} \overline{\bigcup_{\tau \geq t} \pi(\tau, x)}$.

Theorem 6.2. Suppose that the following conditions are fulfilled:

(i) $\mathcal{M}$ is a finite subset of $C(W)$, i.e. $\mathcal{M}:=\left\{f_{1}, f_{2}, \ldots, f_{m}\right\}(m \geq 2)$;

(ii) $\mathcal{M}$ is contracting in the extended sense.

Then the following statement hold:

(i) the skew-product dynamical system $(X, \mathbb{T}, \pi)$ generated by $D I(\mathcal{M})$ is compactly dissipative;

(ii) if every map $f \in \mathcal{M}$ is invertible, then:

(a) the Levinson center $J$ of the skew-product dynamical system $(X, \mathbb{T}, \pi)$ is a chaotic Cantor set;

(b) there exists a residual subset $J_{0} \subseteq J$ (large in the sense of Baire category) consisting of points that are Poisson stable in the positive direction such that the positive semitrajectory of every point $x_{0} \in J_{0}$ is dense on $J$ (i.e. $H\left(x_{0}\right)=J$ for all $\left.x_{0} \in J_{0}\right)$;

(iii) the cocycle $\left\langle W, \varphi,\left(\Omega, \mathbb{Z}_{+}, \sigma\right)\right\rangle\left(\Omega:=C\left(\mathbb{Z}_{+}, \mathcal{M}\right)\right)$ generated by $D I(\mathcal{M})$ is compactly dissipative;

(iv) the Levinson center I of $\varphi$ possesses the following properties:

(a) $I=\overline{\operatorname{Per}(\varphi)}$, where $\operatorname{Per}(\varphi):=\{u \in W: \exists \tau \in \mathbb{N}$ and $\omega \in \Omega$ such that $\sigma(\tau, \omega)=$ $\omega$ and $\varphi(\tau, u, \omega)=u\}$

(b) $I=p r_{1}(J)$, i.e. I is a continuous image of the Cantor set $J$.

Proof. By Theorem 6.1, the cocycle $\varphi$ generated by $D I(\mathcal{M})$ is compactly dissipative and, hence, the skew-product dynamical system $(X, \mathbb{T}, \pi)(X:=W \times \Omega, \pi:=(\varphi, \sigma))$ is compactly dissipative too.

Now we will prove that the Levinson center $J$ of the skew-product dynamical system $(X, \pi)$ possesses the properties listed in the theorem. With this aim, we note that $\Omega:=C\left(\mathbb{Z}_{+}, \mathcal{M}\right)$ $\left(\mathcal{M}:=\left\{f_{1}, f_{2}, \ldots, f_{m}\right\}\right)$ is topologically isomorphic to $\Sigma_{m}:=\{0,1, \ldots, m-1\}^{\mathbb{Z}_{+}}$and, consequently (see, for example, [28,35]), we have:

(i) $\Omega$ is a Cantor set;

(ii) $\overline{\operatorname{Per}(\sigma)}=\Omega$;

(iii) there exists a residual subset $\Omega_{0} \subset \Omega$ consisting of points that are Poisson stable in the positive direction such that $H^{+}\left(\omega_{0}\right)=\Omega$ for all $\omega_{0} \in \Omega_{0}$.

By Theorem 4.4 the Levinson center $J$ of the skew-product dynamical system $\left(X, \mathbb{Z}_{+}, \pi\right)$ is dynamically isomorphic to $(\Omega, \mathbb{T}, \sigma)$ and, consequently, it possesses the following properties:

(i) $J$ is a Cantor set;

(ii) $\overline{\operatorname{Per}(\pi)}=J$; 
(iii) there exists a residual subset $J_{0} \subseteq J$ consisting of points that are Poisson stable in the positive direction such that the positive semi-trajectory of every point $x_{0} \in J_{0}$ is dense on $J$.

Now let $I$ be the Levinson center of the cocycle $\varphi$. According to Theorem 6.1, $I=\overline{\operatorname{Per}(\varphi)}$ and, by Theorem 5.7, $I=\operatorname{pr}_{1}(J)$.

Remark 6.3. The problem of the existence of compact global attractors for $D I(\mathcal{M})$ with finite $\mathcal{M}$ (a collage or iterated function system (IFS)) was studied before in the works $[3,6-8,18]$ (see also the bibliography therein). In $[3,6-8,18]$ the statement close to Theorem 6.2 was proved. Namely:

(i) in [3] the first statement of Theorem 6.2 was announced and the second statement proved;

(ii) in $[6-8,18]$ they considered the case where $W$ is a compact metric space and every map $f \in \mathcal{M}=\left\{f_{1}, f_{2}, \ldots, f_{m}\right\}(i=1, \ldots, m)$ is contracting (not obligatory invertible). For this type of $D I(\mathcal{M})$ they proved the existence of a compact global attractor $\mathcal{A}$ such that for all $u \in \mathcal{A}$ and almost all $\omega \in \Omega$ (with respect to a certain measure on $\Omega$ ) the trajectory $\varphi(n, u, \omega)=U(n, \omega) u\left(U(n, \omega):=\prod_{k=0}^{n} f_{i_{k}},\left(i_{k} \in\{1, \ldots, m\}\right)\right.$ and $\left.f_{i_{0}}:=I d_{W}\right)$ was dense in $\mathcal{A}$.

\section{Acknowledgments}

The authors would like to thank the anonymous referees for their comments and suggestions regarding a preliminary version of this article.

\section{References}

[1] V.M. Alekseev, Symbolic Dynamics, Naukova Dumka, Kiev, 1986. The 11th Mathematical School.

[2] M. Artzrouni, On the convergence of infinite products of matrices, Linear Algebra and its Applications 74 (1986) 11-21.

[3] M.F. Barnsley, Fractals Everywhere, Academic Press, New York, 1988.

[4] M.F. Barnsley, A.D. Sloan, A better way to compress images, BYTE (13) (1988) 215-223.

[5] W.-J. Beyn, L. Elsner, Infinite products and paracontracting matrices, The Electronic Journal of Linear Algebra 2 (1997) 1-8.

[6] N.A. Bobylev, S.V. Emel'yanov, S.K. Korovin, Attractors of discrete controlled systems in metric spaces, Computational Mathematics and Modeling 11 (4) (2000) 321-326. Translated from Prikladnaya Mathematika i Informatika (3) (1999) 5-10.

[7] N.A. Bobylev, A.Yu. Zalozhnev, A.Yu. Klykov, Attractors of ensembles of dynamical systems, Automation and Remote Control 60 (2) (1999) 149-155. Translated from Avtomatika i Telemekhanika (2) (1999) 3-11.

[8] V.A. Bondarenko, V.L. Dolnikov, Fractal image compression by the Barnsley-Sloan method, Automation and Remote Control 55 (5) (1994) 623-629. Translated from Avtomatika i Telemekhanika (5) (1994) 12-20.

[9] N. Bourbaki, Variétés Différentielles et Analitiques (Fascicule de résultats), Herman, Paris, 1971.

[10] R. Bru, L. Elsner, M. Neumann, Convergence of infinite products of matrices and inner-outer iteration schemes, Electronic Transactions on Numerical Analysis 2 (1994) 183-194.

[11] D.N. Cheban, D.S. Fakeeh, Global Attractors of Disperse Dynamical Systems. Sigma, Chişinău, 1994.

[12] D.N. Cheban, Global Attractors of Nonautonomous Dissipative Dynamical Systems. Interdisciplinary Mathematical Sciences 1, World Scientific, River Edge, NJ, 2004, p. 528.

[13] D.N. Cheban, C. Mammana, Asymptotic Stability of Autonomous and Non-Autonomous Discrete Linear Inclusions, Bulletinul Academiei de Stiinte a Republicii Moldova. Matematica 3 (46) (2004) 41-52.

[14] D.N. Cheban, C. Mammana, Absolute Asymptotic Stability of Discrete Linear Inclusions, Bulletinul Academiei de Stiinte a Republicii Moldova. Matematica 1 (47) (2005) 43-68.

[15] I. Daubechies, J.C. Lagarias, Sets of matrices all infinite products of which converge, Linear Algebra and its Applications 161 (1992) 227-263. 
[16] L. Elsner, S. Friedland, Norm conditions for convergence of infinite products, Linear Algebra and its Applications 250 (1997) 133-142.

[17] L. Elsner, I. Koltracht, M. Neumann, On the convergence of asynchronous paracontractions with applications to tomographic reconstruction from incomplete data, Linear Algebra and its Applications 130 (1990) 65-82.

[18] S.V. Emel'yanov, S.K. Korovin, N.A. Bobylev, Attractors of control systems, Doklady Mathematics 61 (1) (2000) 91-94.

[19] L. Gurvits, Geometrical Approach to Some Problems of Control, Observation and Lumping, Ph.D. Thesis, Dept. of Mathematics, Gorky State Univ., USSR, 1985.

[20] L.N. Gurvits, A.M. Zaharin, System theoretic and graph theoretic aspects of Markov chains lumping, in: 5th International Conference on Mathematical Statistics, Vilnius, 1989.

[21] L.N. Gurvits, A.M. Zaharin, Lumpability of Markov processes and systems theory, in: MTNS, Amsterdam, 1989.

[22] L. Gurvits, Stability of discrete linear inclusion, Linear Algebra and its Applications 231 (1995) 47-85.

[23] D. Husemoller, Fibre Bundles, Springer-Verlag, New York, Heidelberg, Berlin, 1994.

[24] J.L. Kelley, General Topology, in: Graduate Texts in Mathematics, vol. 27, Springer-Verlag, Berlin, Heidelberg, New York, 1975.

[25] V.S. Kozyakin, Algebraic insolvability of problem of absolute stability of desynchronized systems, Automation and Remote Control 51 (6) (1990) 754-759.

[26] A.P. Molchanov, Lyapunov function for nonlinear discrete-time control systems, Avtomatika i Telemekhanika 6 (1987) 26-35.

[27] V.I. Opoitsev, Stability of Non-Autonomous Systems, Avtomatika i Telemekhanika (10) (1986) 56-63.

[28] C. Robinson, Dynamical Systems: Stability, Symbolic Dynamics and Chaos, in: Studies in Advanced Mathematics, CRC Press, Boca Raton, Florida, 1995.

[29] G.R. Sell, Topological Dynamics and Ordinary Differential Equations, Van Nostrand-Reinhold, London, 1971.

[30] B.A. Shcherbakov, Topological Dynamics and Poisson's Stability of Solutions of Differential Equations, Kishinev, Shtiintsa, 1972 (in Russian).

[31] K.S. Sibirskii, A.S. Shube, Semidynamical Systems, Stiintsa, Kishinev 1987 (in Russian).

[32] A.N. Sharkovsky, Yu.L. Maistrenko, E.Yu. Romanenko, Difference Equations and Their Applications, Kluwer Academic Publisher, Dordrecht, Boston, London, 1993.

[33] J. Stark, Invariant graphs for forced systems, Physica D 109 (1997) 163-179.

[34] A. Vladimirov, L. Elsner, W.-J. Beyn, Stability and paracontractivity of discrete linear inclusion, Linear Algebra and its Applications 312 (2000) 125-134.

[35] J. de Vries, Elements of Topological Dynamics, in: Mathematics and Its Applications, Vol. 257, Kluwer Academic Publishers, Dordrecht, Boston, London, 1993.

[36] F. Wirth, On stability of infinite-dimensional discrete inclusions, Journal of Mathematical Systems, Estimations, and Control 8 (4) (1998) 507-510. ftp://trick.ntp.springer.de/pub/jmsec/ Retrieval code 72701 (Full electronic manuscript).

[37] F. Wirth, The generalized spectral radius and external norms, Linear Algebra and its Applications 342 (2002) 17-40. 